\title{
Existence and Stability of Periodic Solution to Delayed Nonlinear Differential Equations
}

\author{
Xiang Gu, ${ }^{1}$ Huicheng Wang, ${ }^{1}$ P. J. Y. Wong, ${ }^{2}$ and Yonghui Xia ${ }^{1}$ \\ ${ }^{1}$ Department of Mathematics, Zhejiang Normal University, Jinhua, Zhejiang 321004, China \\ ${ }^{2}$ School of Electrical and Electronic Engineering, Nanyang Technological University, Singapore 639798 \\ Correspondence should be addressed to Yonghui Xia; yhxia@zjnu.cn
}

Received 23 February 2014; Accepted 17 March 2014; Published 14 April 2014

Academic Editor: Yongli Song

Copyright (c) 2014 Xiang Gu et al. This is an open access article distributed under the Creative Commons Attribution License, which permits unrestricted use, distribution, and reproduction in any medium, provided the original work is properly cited.

The main purpose of this paper is to study the periodicity and global asymptotic stability of a generalized Lotka-Volterra's competition system with delays. Some sufficient conditions are established for the existence and stability of periodic solution of such nonlinear differential equations. The approaches are based on Mawhin's coincidence degree theory, matrix spectral theory, and Lyapunov functional.

\section{Introduction and Motivation}

In the past few decades, differential equations have been used in the study of population dynamics, ecology and epidemiology, malaria transmission, and so forth (see, e.g., [1-10]). One of the rudimentary population systems is the nonautonomous $n$-species competitive model:

$$
\dot{y}_{i}(t)=y_{i}(t)\left[b_{i}(t)-\sum_{j=1}^{n} a_{i j}(t) y_{j}(t)\right], \quad i=1,2, \ldots, n .
$$

Based on Mawhin's coincidence degree theory, spectral theory, and novel estimation techniques for the priori bounds of unknown solutions to the equation $L x=\lambda N x$, Xia and Han [8] studied the existence and stability of periodic solution for (1). But model (1) is doubted by Gilpin and Ayala [11], they thought that the model is not reasonable enough. In order to fit data in the experiments conducted in Ayala et al. [12] and to yield significantly more accurate results on the competitive model, Chen [13] proposed a more complicated model as follows:

$$
\dot{y}_{i}(t)=y_{i}(t)\left[r_{i}(t)-\sum_{j=1}^{n} a_{i j}(t) y_{j}^{\alpha_{i j}}(t)\right], \quad i=1,2, \ldots, n,
$$

where $\alpha_{i}$ provides a nonlinear measure of intraspecific interference and $\alpha_{i j}$ provides a measure of interspecific interference. Chen studied the permanence of (2) by average method. For the sake of convenience, in what follows, the new factor introduced by Gilpin and Ayala is called GilpinAyala effect. On the other hand, many scholars think that the delayed models are more realistic. Because time delays may lead to oscillation, bifurcation, chaos, and instability which may be harmful to a system. In fact, May [14] has shown that if a time delay is incorporated into the resource limitation of the logistic equation, then it has destabilizing effect on the stability of the system (also see Cooke and Grossman [15]). But sometimes, the delays may be harmless under some restriction and this is more important in some sense (e.g., see [16]). A very basic and important ecological problem in the study of multispecies population dynamics concerns the global existence and global asymptotic stability of positive 
periodic solutions. It is doubted whether the existence and stability of periodic solutions can be affected by the delays or Gilpin-Ayala effect. For this reason, in the present paper, we consider the Gilpin-Ayala type delayed system as follows:

$$
\begin{aligned}
\dot{y}_{i}(t)=y_{i}(t) & {\left[r_{i}(t)-\sum_{j=1}^{n} a_{i j}(t) y_{j}^{\alpha_{i j}}(t)\right.} \\
& \left.-\sum_{j=1}^{n} b_{i j}(t) y_{j}^{\beta_{i j}}\left(t-\tau_{i j}\right)\right], \quad i=1,2, \ldots, n,
\end{aligned}
$$

where $y_{i}$ is the population density of the $i$ th species; $r_{i}$ is the intrinsic exponential growth rate of the $i$ th species; $a_{i j}, b_{i j}$ measure the amount of competition between the $i$ th species and the $j$ th species $(i \neq j)$; and $\alpha_{i j}, \beta_{i j}$ provide a nonlinear measure of intraspecific interference. For the point of biological view, the coefficients are assumed to be continuous $\omega$-periodic functions; we always assume that $r_{i}$, $a_{i j}, b_{i j}, \tau_{i j}, i, j=1,2, \ldots, n$, are nonnegative and $a_{i i}, b_{i i}$ are strictly positive. And system (3) is supplemented with the initial condition

$$
y_{i}(s)=\phi_{i}(s), \quad s \in[-\tau, 0], \phi_{i}(0)>0, i=1,2, \ldots, n,
$$

where $\tau=\max _{1 \leq i \leq n}\left\{\tau_{i j}\right\}, \phi=\left(\phi_{1}, \ldots, \phi_{n}\right) \in \mathrm{BC}\left([-\tau, 0], \mathbf{R}_{+}^{n}\right)$, and $\mathrm{BC}$ is the set of all bounded continuous functions from $[-\tau, 0]$ into $\mathbf{R}_{+}^{n}$. It is easy to see that for such given initial value condition, the corresponding solution of (3) remains positive for all $t \geq 0$. The purpose of this paper is to obtain some new and interesting criteria for the existence and global asymptotic stability of periodic solution of system (3).

The structure of this paper is as follows. In Section 2, some new and interesting sufficient conditions for the existence of periodic solution of system (3) are obtained. Section 3 is devoted to examining the stability of this periodic solution. In Section 4, some corollaries and discussion are presented. Finally, some examples and their simulations are given to show the effectiveness and feasibility of our results.

\section{Existence of Periodic Solutions}

In this section, we will obtain some sufficient conditions for the existence of periodic solution of system (3).

2.1. Preliminaries on the Matrix Theory and Degree Theory. For convenience, we introduce some notations, definitions, and lemmas. If $f(t)$ is a continuous $\omega$-periodic function defined on $\mathbf{R}$, denote

$$
\begin{gathered}
\underline{f}=\min _{t \in[0, \omega]}|f(t)|, \quad \bar{f}=\max _{t \in[0, \omega]}|f(t)|, \\
m(f)=\frac{1}{\omega} \int_{0}^{\omega} f(t) d t .
\end{gathered}
$$

We use $x=\left(x_{1}, \ldots, x_{n}\right)^{T} \in \mathbf{R}^{n}$ to denote a column vector, $\mathscr{D}=\left(d_{i j}\right)_{n \times n}$ is an $n \times n$ matrix, $\mathscr{D}^{T}$ denotes the transpose of
$\mathscr{D}$, and $E_{n}$ is the identity matrix of size $n$. A matrix or vector $\mathscr{D}>0$ (resp., $\mathscr{D} \geq 0$ ) means that all entries of $\mathscr{D}$ are positive (resp., nonnegative). For matrices or vectors $\mathscr{D}$ and $E, \mathscr{D}>E$ (resp., $\mathscr{D} \geq E$ ) means that $\mathscr{D}-E>0$ (resp., $\mathscr{D}-E \geq 0$ ). We also denote the spectral radius of the matrix $\mathscr{D}$ by $\rho(\mathscr{D})$.

If $v=\left(v_{1}, v_{2}, \ldots, v_{n}\right)^{T} \in \mathbf{R}^{n}$, then we have a choice of vector norms in $\mathbf{R}^{n}$; for instance, $\|v\|_{1},\|v\|_{2}$, and $\|v\|_{\infty}$ are the commonly used norms, where

$$
\begin{aligned}
& \|v\|_{1}=\sum_{j=1}^{n}\left|v_{i}\right|, \\
& \|v\|_{2}=\left\{\sum_{j=1}^{n}\left|v_{i}\right|^{2}\right\}^{1 / 2}, \\
& \|v\|_{\infty}=\max _{1 \leq i \leq n}\left|v_{i}\right| .
\end{aligned}
$$

We recall the following norms of matrices induced by respective vector norms. For instance, if $\mathscr{A}=\left(a_{i j}\right)_{n \times n}$, the norm of the matrix $\|\mathscr{A}\|$ induced by a vector norm $\|\cdot\|$ is defined by

$$
\|\mathscr{A}\|_{p}=\sup _{v \in \mathbf{R}^{n}, v \neq 0} \frac{\|\mathscr{A} v\|_{p}}{\|v\|_{p}}=\sup _{\|v\|_{p}=1}\|\mathscr{A} v\|_{p}=\sup _{\|v\|_{p} \leq 1}\|\mathscr{A} v\|_{p}
$$

In particular one can show that $\|\mathscr{A}\|_{1}=\max _{1 \leq j \leq n} \sum_{i=1}^{n}\left|a_{i j}\right|$ (column norm) and $\|\mathscr{A}\|_{2}=\left[\lambda_{\max }\left(\mathscr{A}^{T} \mathscr{A}\right)\right]^{1 / 2}=[\max$. eigenvalue of $\left.\left(\mathscr{A}^{T} \mathscr{A}\right)\right]^{1 / 2},\|\mathscr{A}\|_{\infty}=\max _{1 \leq i \leq n} \sum_{j=1}^{n}\left|a_{i j}\right|$ (row norm).

Definition 1 (see $[17,18]$ ). Let $X, Z$ be normed real Banach spaces, let $L:$ Dom $L \subset X \rightarrow Z$ be a linear mapping, and let $N: X \rightarrow Z$ be a continuous mapping. The mapping $L$ is called a Fredholm mapping of index zero if $\operatorname{dim} \operatorname{Ker} L=$ codim $\operatorname{Im} L<+\infty$ and $\operatorname{Im} L$ is closed in $Z$. If $L$ is a Fredholm mapping of index zero and there exist continuous projectors $P: X \rightarrow X$ and $Q: Z \rightarrow Z$ such that $\operatorname{Im} P=\operatorname{Ker} L$ and $\operatorname{Ker} Q=\operatorname{Im} L=\operatorname{Im}(I-Q)$, it follows that $L \mid \operatorname{dom} L \cap \operatorname{Ker} P$ : $(I-P) X \rightarrow \operatorname{Im} L$ is invertible. We denote the inverse of that map by $K_{P}$. If $\Omega$ is an open bounded subset of $X$, the mapping $N$ will be called $L$-compact on $\bar{\Omega}$ if $Q N(\bar{\Omega})$ is bounded and $K_{P}(I-Q) N: \bar{\Omega} \rightarrow X$ is compact. Since $\operatorname{Im} Q$ is isomorphic to $\operatorname{Ker} L$, there exists an isomorphism $J: \operatorname{Im} Q \rightarrow \operatorname{Ker} L$.

Definition 2 (see $[17,18]$ ). Let $\Omega \subset \mathbf{R}^{n}$ be open and bounded, $f \in C^{1}\left(\Omega, \mathbf{R}^{n}\right) \cap C\left(\bar{\Omega}, \mathbf{R}^{n}\right)$, and $y \in \mathbf{R}^{n} / f\left(\partial \Omega \cup N_{f}\right)$; that is, $y$ is a regular value of $f$. Here, $N_{f}=\left\{x \in \Omega: J_{f}(x)=0\right\}$, the critical set of $f$, and $J_{f}$ is the Jacobian of $f$ at $x$. Then the degree $\operatorname{deg}\{f, \Omega, y\}$ is defined by

$$
\operatorname{deg}\{f, \Omega, y\}=\sum_{x \in f^{-1}(y)} \operatorname{sgn} J_{f}(x)
$$

with the agreement that $\sum \phi=0$. For more details about degree theory, the reader is referred to [18]. 
Lemma 3 (continuation theorem [17]). Let $\Omega \subset X$ be an open and bounded set. Let $L$ be a Fredholm mapping of index zero and let $N$ be L-compact on $\bar{\Omega}$ (i.e., $Q N(\bar{\Omega})$ is bounded and $K_{P}(I-Q) N: \bar{\Omega} \rightarrow X$ is compact). Assume,

(i) for each $\lambda \in(0,1), x \in \partial \Omega \cap \operatorname{Dom} L$ and $L x \neq \lambda N x$;

(ii) for each $x \in \partial \Omega \cap \operatorname{Ker} L, Q N x \neq 0$ and $\operatorname{deg}\{J Q N, \Omega \cap$ $\operatorname{Ker} L, 0\} \neq 0$.

Then $L x=N x$ has at least one solution in $\bar{\Omega} \cap \operatorname{Dom} L$.

Definition 4 (see $[19,20])$. A real $n \times n$ matrix $\mathscr{A}=\left(a_{i j}\right)$ is said to be an $M$-matrix if $a_{i j} \leq 0, i, j=1,2, \ldots, n, i \neq j$, and $\mathscr{A}^{-1} \geq 0$.

Lemma 5 (see $[19,20])$. Let $\mathscr{A} \geq 0$ be an $n \times n$ matrix and $\rho(\mathscr{A})<1$; then $\left(E_{n}-\mathscr{A}\right)^{-1} \geq 0$, where $E_{n}$ denotes the identity matrix of size $n$.

In what follows, we will introduce some function spaces and their norms, which are valid throughout this paper. Denote

$$
\begin{gathered}
X=\left\{x(t)=\left(x_{1}(t), x_{2}(t), \ldots, x_{n}(t)\right)^{T}\right. \\
\left.\quad \in C^{1}\left(\mathbf{R}, \mathbf{R}^{n}\right) \mid x(t+\omega)=x(t) \forall t \in \mathbf{R}\right\}, \\
Z=\left\{x(t)=\left(x_{1}(t), x_{2}(t), \ldots, x_{n}(t)\right)^{T}\right. \\
\left.\in C\left(\mathbf{R}, \mathbf{R}^{n}\right) \mid x(t+\omega)=x(t) \forall t \in \mathbf{R}\right\} .
\end{gathered}
$$

And, the norms are given by

$$
\begin{aligned}
& \left|x_{i}(t)\right|_{0}=\max _{t \in[0, \omega]}\left|x_{i}(t)\right|, \quad\left|x_{i}(t)\right|_{1}=\left|x_{i}(t)\right|_{0}+\left|\dot{x}_{i}(t)\right|_{0} \\
& i=1,2, \ldots, n, \\
& \|x(t)\|_{0}=\max _{1 \leq i \leq n}\left\{\left|x_{i}(t)\right|_{0}\right\}, \\
& \|x(t)\|_{1}=\|x(t)\|_{0}+\|\dot{x}(t)\|_{0}=\max _{1 \leq i \leq n}\left\{\left|x_{i}(t)\right|_{1}\right\} .
\end{aligned}
$$

Obviously, $X$ and $Z$, respectively, endowed with the norms $\|\cdot\|_{1}$ and $\|\cdot\|_{0}$ are Banach spaces.

\subsection{Result on the Existence of Periodic Solutions}

Theorem 6. Assume that the following conditions hold:

$\left(\mathrm{H}_{1}\right)$ the system of algebraic equations

$$
f(u):=\left(m\left(r_{i}\right)-\sum_{j=1}^{n} m\left(a_{i j}\right) u_{j}^{\alpha_{i j}}-\sum_{j=1}^{n} m\left(b_{i j}\right) u_{j}^{\beta_{i j}}\right)_{n \times 1}=0
$$

has finite solutions $\left(u_{1}^{*}, u_{2}^{*}, \ldots, u_{n}^{*}\right)^{T} \in \mathbf{R}_{+}^{n}$ with $u_{i}^{*}>0$ and $\sum_{u^{*}} \operatorname{sgn} J_{f}\left(u^{*}\right) \neq 0$;

$\left(\mathrm{H}_{2}\right) \alpha_{j i} \leq \alpha_{i i}, \beta_{j i} \leq \alpha_{i i}(j \neq i), \alpha_{i i}=\beta_{i i}, \tau_{i i} \equiv 0, i, j=$ $1,2, \ldots, n$;
$\left(\mathrm{H}_{3}\right) \rho(\mathscr{K})<1$, where $\mathscr{K}=\left(\Gamma_{i j}\right)_{n \times n}$ and

$$
\Gamma_{i j}= \begin{cases}0, & i=j, \\ \frac{\left(\bar{a}_{i j}+\bar{b}_{i j}\right)}{\left(\underline{a}_{j j}+\underline{b}_{j j}\right)}, & i \neq j .\end{cases}
$$

Then system (3) has at least one positive w-periodic solution.

Proof. Note that every solution $y(t)=\left(y_{1}(t), y_{2}(t), \ldots\right.$, $\left.y_{n}(t)\right)^{T}$ of system (3) with the initial value condition is positive. Make the change of variables

$$
y_{i}(t)=e^{x_{i}(t)}, \quad i=1,2, \ldots, n .
$$

Then system (3) is the same as

$$
\begin{aligned}
\dot{x}_{i}(t)= & r_{i}(t)-\sum_{j=1}^{n} a_{i j}(t) e^{\alpha_{i j} x_{j}(t)} \\
& -\sum_{j=1}^{n} b_{i j}(t) e^{\beta_{i j} x_{j}\left(t-\tau_{i j}\right)}, \quad i=1,2, \ldots, n .
\end{aligned}
$$

Obviously, system (3) that has at least one $\omega$-periodic solution is equivalent to system (14) that has at least one $\omega$-periodic solution. To prove Theorem 6 , our main tasks are to construct the operators (i.e., $L, N, P$, and $Q$ ) appearing in Lemma 3 and to find an appropriate open set $\Omega$ satisfying conditions (i) and (ii) in Lemma 3. To this end, we proceed with three steps.

Step 1. In this step, we intend to construct the operators appearing in Lemma 3 and verify that they satisfy the conditions of Lemma 3. For any $x(t) \in X$, in view of the periodicity, it is easy to check that

$$
\begin{aligned}
\Delta_{i}(x, t)= & r_{i}(t)-\sum_{j=1}^{n} a_{i j}(t) e^{\alpha_{i j} x_{j}(t)} \\
& -\sum_{j=1}^{n} b_{i j}(t) e^{\beta_{i j} x_{j}\left(t-\tau_{i j}\right)} \in Z .
\end{aligned}
$$

And define the operators $L: \operatorname{Dom} L \subset X \rightarrow Z$ and $N$ : $X \rightarrow Z$ as follows:

$$
\begin{aligned}
X \ni x(t) & \longrightarrow(L x)(t)=\frac{d x(t)}{d t} \in Z, \\
X \ni x(t) & \longrightarrow(N x)(t) \\
& =\left((N x)_{1}(t),(N x)_{2}(t), \ldots,(N x)_{n}(t)\right)^{T} \in Z,
\end{aligned}
$$

where

$$
(N x)_{i}(t)=\Delta_{i}(x, t), \quad i=1,2, \ldots, n .
$$


Define, respectively, the projectors $P: X \rightarrow X$ and $Q: Z \rightarrow$ $Z$ by

$$
\begin{array}{r}
P x(t)=\frac{1}{\omega} \int_{0}^{\omega} x(t) d t, \quad Q z(t)=\frac{1}{\omega} \int_{0}^{\omega} z(t) d t, \\
x \in X, z \in Z .
\end{array}
$$

It can be found that the domain of $L$ in $X$ is actually the whole space, and

$$
\begin{aligned}
& \operatorname{Ker} L=\{x(t) \in X \mid L x(t)=0, \text { i.e., } \dot{x}(t)=0\}=\mathbf{R}^{n}, \\
& \operatorname{Im} L=\left\{z(t) \in Z \mid \int_{0}^{\omega} z(t) d t=0\right\} \text { is closed in } Z .
\end{aligned}
$$

Moreover, $P, Q$ are continuous operators such that

$$
\begin{aligned}
\operatorname{Im} P= & \mathbf{R}^{n}=\operatorname{Ker} L, \quad \operatorname{Im} L=\operatorname{Ker} Q=\operatorname{Im}(I-Q), \\
& \operatorname{dim} \operatorname{Ker} L=\text { codim } \operatorname{Im} L=n<+\infty .
\end{aligned}
$$

It follows that $L$ is a Fredholm mapping of index zero. Furthermore, the generalized inverse (to $L$ ) $K_{P}: \operatorname{Im} L \rightarrow$ Dom $L \cap \operatorname{Ker} P$ exists, which is given by

$$
K_{P}(y)=\int_{0}^{t} y(s) d s-\frac{1}{\omega} \int_{0}^{\omega} \int_{0}^{t} y(s) d s d t .
$$

Then $Q N: X \rightarrow Z$ and $K_{P}(I-Q) N: X \rightarrow X$ are defined by

$$
\begin{aligned}
\text { QN } x=\left(\frac{1}{\omega} \int_{0}^{\omega} \Delta_{1}(x, t) d t, \frac{1}{\omega} \int_{0}^{\omega} \Delta_{2}(x, t) d t, \ldots,\right. \\
\left.\frac{1}{\omega} \int_{0}^{\omega} \Delta_{n}(x, t) d t\right)^{T},
\end{aligned}
$$

$$
K_{P}(I-Q) N x=\left(\Psi_{1}(x, t), \Psi_{2}(x, t), \ldots, \Psi_{n}(x, t)\right)^{T},
$$

where

$$
\begin{aligned}
\Psi_{k}(x, t)= & \int_{0}^{t} \Delta_{k}(x, s) d s-\frac{1}{\omega} \int_{0}^{\omega} \int_{0}^{t} \Delta_{k}(x, s) d s d t \\
& -\left(\frac{t}{\omega}-\frac{1}{2}\right) \int_{0}^{\omega} \Delta_{k}(x, s) d s, \quad k=1,2, \ldots, n .
\end{aligned}
$$

Clearly, $Q N$ and $K_{P}(I-Q) N$ are continuous. Now we turn to show the fact that for any open bounded set $\Omega \subset X$, denoted by

$$
\Omega=\left\{\left.x(t) \in X|| x_{i}(t)\right|_{1}=\left|x_{i}(t)\right|_{0}+\left|\dot{x}_{i}(t)\right|_{0}<h_{i}\right\},
$$

the mapping $N$ is $L$-compact on $\bar{\Omega}$. Here, the constants $h_{i}$ are independent of the choice of $x(t)$. In view of Definition 1 , to show the above fact, it suffices to show that $Q N(\bar{\Omega})$ is bounded and $K_{P}(I-Q) N: \bar{\Omega} \rightarrow X$ is compact. We first arrive at

$$
\begin{array}{r}
\left|(Q N x)_{i}\right|_{0}=\left|\frac{1}{\omega} \int_{0}^{\omega} \Delta_{i}(x, t) d t\right|_{0} \leq\left|\Delta_{i}(x, t)\right|_{0}:=M_{i}, \\
\forall x \in \bar{\Omega},
\end{array}
$$

which implies that $Q N(\bar{\Omega})$ is bounded in the space $\left(Z,\|\cdot\|_{0}\right)$. Secondly, we will show that $\left(K_{P}(I-Q) N x\right)(\bar{\Omega})$ is relatively compact in the space $\left(X,\|\cdot\|_{1}\right)$. In fact, it follows from (22) that

$$
\left(K_{P}(I-Q) N x\right)^{\prime}=\left(\Psi_{1}^{\prime}(x, t), \Psi_{2}^{\prime}(x, t), \ldots, \Psi_{n}^{\prime}(x, t)\right)^{T},
$$

where $I=d / d t$ and

$$
\Psi_{k}^{\prime}(x, t)=\Delta_{k}(x, t)-\frac{1}{\omega} \int_{0}^{\omega} \Delta_{k}(x, s) d s, \quad k=1,2, \ldots, n .
$$

This, combining with (22), gives

$$
\begin{aligned}
\left|\left(K_{P}(I-Q) N x\right)_{i}(t)\right|_{1} \\
=\left|\left(K_{P}(I-Q) N x\right)_{i}(t)\right|_{0}+\left|\left(K_{P}(I-Q) N x\right)_{i}^{\prime}(t)\right|_{0} \\
\leq M_{i} \omega+\frac{1}{2} M_{i} \omega+\frac{1}{2} M_{i} \omega \\
\quad+M_{i}+M_{i}=2(\omega+1) M_{i},
\end{aligned}
$$

which implies that $K_{P}(I-Q) N(\bar{\Omega})$ is bound in the space $\left(X,\|\cdot\|_{1}\right)$.

On the other hand, we prove that $\left(K_{P}(I-Q) N x\right)(\bar{\Omega})$ is equicontinuous. In view of uniform continuity of $r_{i}, a_{i j}$, and $b_{i j}$, for any $\varepsilon>0$, there exists $\delta_{1}>0$ such that, for any $t, s \in \mathbf{R}$, provided that $|t-s|<\delta_{1}$, we have

$$
\begin{gathered}
\left|r_{i}(t)-r_{i}(s)\right|<\varepsilon, \quad\left|a_{i j}(t)-a_{i j}(s)\right|<\varepsilon, \\
\left|b_{i j}(t)-b_{i j}(s)\right|<\varepsilon .
\end{gathered}
$$

Since any $x(t)=\left(x_{1}(t), x_{2}(t), \ldots, x_{n}(t)\right)^{T} \in \bar{\Omega}$ is equicontinuous, for the same $\varepsilon$, there exists $0<\delta_{2} \leq \delta_{1}$ such that, for any $t, s \in \mathbf{R}$, provided that $|t-s|<\delta_{2}$, we have

$$
\left|x_{i}(t)-x_{i}(s)\right|_{0}<\varepsilon .
$$

It follows from (29) and (30) that

$$
\begin{aligned}
& \left|\Delta_{i}(x(t), t)-\Delta_{i}(x(s), s)\right|_{0} \\
& =\mid r_{i}(t)-\sum_{j=1}^{n} a_{i j}(t) e^{\alpha_{i j} x_{j}(t)}-\sum_{j=1}^{n} b_{i j}(t) e^{\beta_{i j} x_{j}\left(t-\tau_{i j}\right)} \\
& \quad-r_{i}(s)+\sum_{j=1}^{n} a_{i j}(s) e^{\alpha_{i j} x_{j}(s)}+\left.\sum_{j=1}^{n} b_{i j}(s) e^{\beta_{i j} x_{j}\left(s-\tau_{i j}\right)}\right|_{0} \\
& \leq\left|r_{i}(t)-r_{i}(s)\right|_{0}+\sum_{j=1}^{n}\left|a_{i j}(t) e^{\alpha_{i j} x_{j}(t)}-a_{i j}(s) e^{\alpha_{i j} x_{j}(s)}\right|_{0} \\
& \quad+\sum_{j=1}^{n}\left|b_{i j}(t) e^{\beta_{i j} x_{j}\left(t-\tau_{i j}\right)}-b_{i j}(s) e^{\beta_{i j} x_{j}\left(s-\tau_{i j}\right)}\right|_{0}
\end{aligned}
$$




$$
\begin{aligned}
& \leq\left|r_{i}(t)-r_{i}(s)\right|+\sum_{j=1}^{n}\left|a_{i j}(t)\right|_{0}\left|e^{\alpha_{i j} x_{j}(t)}-e^{\alpha_{i j} x_{j}(s)}\right|_{0} \\
& +\sum_{j=1}^{n}\left|a_{i j}(t)-a_{i j}(s)\right|_{0}\left|e^{\alpha_{i j} x_{j}(s)}\right|_{0} \\
& +\sum_{j=1}^{n}\left|b_{i j}(t)\right|_{0}\left|e^{\beta_{i j} x_{j}\left(t-\tau_{i j}\right)}-e^{\beta_{i j} x_{j}\left(s-\tau_{i j}\right)}\right|_{0} \\
& +\sum_{j=1}^{n}\left|b_{i j}(t)-b_{i j}(s)\right|_{0}\left|e^{\beta_{i j} x_{j}\left(s-\tau_{i j}\right)}\right|_{0} \\
& <\varepsilon+\sum_{j=1}^{n} \bar{a}_{i j} e^{\alpha_{i j} h_{j}}\left|x_{i}(t)-x_{i}(s)\right|_{0}+\sum_{j=1}^{n} e^{\alpha_{i j} h_{j}} \mathcal{E} \\
& +\sum_{j=1}^{n} \bar{b}_{i j} e^{\beta_{i j} h_{j}}\left|x_{i}\left(t-\tau_{i j}\right)-x_{i}\left(s-\tau_{i j}\right)\right|_{0}+\sum_{j=1}^{n} e^{\beta_{i j} h_{j}} \mathcal{E} \\
& <\varepsilon+\sum_{j=1}^{n} \bar{a}_{i j} e^{\alpha_{i j} h_{j}} \mathcal{\varepsilon}+\sum_{j=1}^{n} e^{\alpha_{i j} h_{j}} \mathcal{\varepsilon}+\sum_{j=1}^{n} \bar{b}_{i j} e^{\beta_{i j} h_{j}} \mathcal{\varepsilon}+\sum_{j=1}^{n} e^{\beta_{i j} h_{j}} \mathcal{\varepsilon} \\
& =\left[1+\sum_{j=1}^{n}\left(1+\bar{a}_{i j}\right) e^{\alpha_{i j} h_{j}}+\sum_{j=1}^{n}\left(1+\bar{b}_{i j}\right) e^{\beta_{i j} h_{j}}\right] \varepsilon .
\end{aligned}
$$

Thus, it follows from (26) that

$$
\begin{aligned}
\left|\left(K_{P}(I-Q) N x\right)_{i}^{\prime}(t)-\left(K_{P}(I-Q) N x\right)_{i}^{\prime}(s)\right|_{0} \\
\quad=\left|\Delta_{i}(x(t), t)-\Delta_{i}(x(s), s)\right|_{0} \\
\quad<\left[1+\sum_{j=1}^{n}\left(1+\bar{a}_{i j}\right) e^{\alpha_{i j} h_{j}}+\sum_{j=1}^{n}\left(1+\bar{b}_{i j}\right) e^{\beta_{i j} h_{j}}\right] \varepsilon .
\end{aligned}
$$

On the other hand, the mean value theorem together with (26) gives

$$
\begin{aligned}
\left.\mid K_{P}(I-Q) N x\right)_{i}(t)-\left.\left(K_{P}(I-Q) N x\right)_{i}(s)\right|_{0} \\
\quad=\left\|\left(K_{P}(I-Q) N x\right)_{i}^{\prime}(\xi)\right\|_{0}|t-s| \leq 2 M_{i}|t-s|,
\end{aligned}
$$

where $\xi$ lies between $t$ and $s$. Taking $\delta=\min \left\{\varepsilon / 2 M_{i}, \delta_{2}\right\}$, it follows from (32) and (33) that $|t-s|<\delta$ implies

$$
\begin{aligned}
\left|\left(K_{P}(I-Q) N x\right)_{i}(t)-\left(K_{P}(I-Q) N x\right)_{i}(s)\right|_{1} \\
=\left|\left(K_{P}(I-Q) N x\right)_{i}(t)-\left(K_{P}(I-Q) N x\right)_{i}(s)\right|_{0} \\
\quad+\left|\left(K_{P}(I-Q) N x\right)_{i}^{\prime}(t)-\left(K_{P}(I-Q) N x\right)_{i}^{\prime}(s)\right|_{0}
\end{aligned}
$$

$$
\begin{aligned}
& =\left[1+\sum_{j=1}^{n}\left(1+\bar{a}_{i j}\right) e^{\alpha_{i j} h_{j}}+\sum_{j=1}^{n}\left(1+\bar{b}_{i j}\right) e^{\beta_{i j} h_{j}}\right] \varepsilon+2 M_{i} \delta \\
& <\left[1+\sum_{j=1}^{n}\left(1+\bar{a}_{i j}\right) e^{\alpha_{i j} h_{j}}+\sum_{j=1}^{n}\left(1+\bar{b}_{i j}\right) e^{\beta_{i j} h_{j}}\right] \varepsilon+\varepsilon \\
& :=\widetilde{M} \varepsilon,
\end{aligned}
$$

which implies that $\left(K_{P}(I-Q) N x\right)(\bar{\Omega})$ is equicontinuous.

Therefore, by the generalized Arzela-Ascoli theorem, we have that $\left(K_{P}(I-Q) N x\right)(\bar{\Omega})$ is relatively compact in the space $\left(X,\|\cdot\|_{1}\right)$. The proof of this step is complete.

Step 2. In this step, we are in a position to search for appropriate open bounded subsets $\Omega$ satisfying condition (i) of Lemma 3. Specifically, our aim is to search for an appropriate $h_{i}$ defined by $\Omega$ in Step 1 such that $\Omega$ satisfies condition (i) of Lemma 3. To this end, assume that $x(t) \in X$ is a solution of the equation $L x=\lambda N x$ for each $\lambda \in(0,1)$; that is,

$$
\begin{array}{r}
\dot{x}_{i}(t)=\lambda\left[r_{i}(t)-\sum_{j=1}^{n} a_{i j}(t) e^{\alpha_{i j} x_{j}(t)}-\sum_{j=1}^{n} b_{i j}(t) e^{\beta_{i j} x_{j}\left(t-\tau_{i j}\right)}\right], \\
i=1,2, \ldots, n .
\end{array}
$$

Since $x(t) \in X$, each $x_{i}(t), i=1,2, \ldots, n$, as components of $x(t)$, is continuously differentiable and $\omega$-periodic. In view of continuity and periodicity, there exists $t_{i} \in[0, \omega]$ such that $x_{i}\left(t_{i}\right)=\max _{t \in[0, \omega]}\left|x_{i}(t)\right|, i=1,2, \ldots, n$. Accordingly, $\dot{x}_{i}\left(t_{i}\right)=$ 0 and we arrive at

$$
\begin{array}{r}
r_{i}\left(t_{i}\right)-\sum_{j=1}^{n} a_{i j}\left(t_{i}\right) e^{\alpha_{i j} x_{j}\left(t_{i}\right)}-\sum_{j=1}^{n} b_{i j}\left(t_{i}\right) e^{\beta_{i j} x_{j}\left(t_{i}-\tau_{i j}\right)}=0, \\
i=1,2, \ldots, n .
\end{array}
$$

That is,

$$
\begin{aligned}
a_{i i}\left(t_{i}\right) e^{\alpha_{i i} x_{i}\left(t_{i}\right)}+b_{i i}\left(t_{i}\right) e^{\beta_{i i} x_{i}\left(t_{i}-\tau_{i i}\right)} & \\
= & r_{i}\left(t_{i}\right)-\sum_{j=1, j \neq i}^{n} a_{i j}\left(t_{i}\right) e^{\alpha_{i j} x_{j}\left(t_{i}\right)} \\
& -\sum_{j=1, j \neq i}^{n} b_{i j}\left(t_{i}\right) e^{\beta_{i j} x_{j}\left(t_{i}-\tau_{i j}\right)}, \quad i=1,2, \ldots n .
\end{aligned}
$$

Noticing that $x_{j}\left(t_{j}\right)=\max _{t \in[0, \omega]}\left|x_{j}(t)\right|$ implies

$$
x_{j}\left(t_{i}\right) \leq x_{j}\left(t_{j}\right), \quad x_{j}\left(t_{i}-\tau_{i j}\right) \leq x_{j}\left(t_{j}\right) .
$$

It follows from $\left(\mathrm{H}_{2}\right)$ and (37) that

$$
\begin{aligned}
& \underline{a}_{i i} e^{\alpha_{i i} x_{i}\left(t_{i}\right)}+\underline{b}_{i i} e^{\alpha_{i i} x_{i}\left(t_{i}\right)} \\
& \quad \leq\left|a_{i i}\left(t_{i}\right) e^{\alpha_{i i} x_{i}\left(t_{i}\right)}+b_{i i}\left(t_{i}\right) e^{\alpha_{i i} x_{i}\left(t_{i}\right)}\right| \\
& \quad \leq\left|a_{i i}\left(t_{i}\right) e^{\alpha_{i i} x_{i}\left(t_{i}\right)}+b_{i i}\left(t_{i}\right) e^{\beta_{i i} x_{i}\left(t_{i}-\tau_{i i}\right)}\right|
\end{aligned}
$$




$$
\begin{aligned}
& \mid r_{i}\left(t_{i}\right)-\sum_{j=1, j \neq i}^{n} a_{i j}\left(t_{i}\right) e^{\alpha_{i j} x_{j}\left(t_{i}\right)} \\
& -\sum_{j=1, j \neq i}^{n} b_{i j}\left(t_{i}\right) e^{\beta_{i j} x_{j}\left(t_{i}-\tau_{i j}\right)} \mid \\
\leq & \bar{r}_{i}+\sum_{j=1, j \neq i}^{n} \bar{a}_{i j} e^{\alpha_{i j} x_{j}\left(t_{i}\right)}+\sum_{j=1, j \neq i}^{n} \bar{b}_{i j} e^{\beta_{i j} x_{j}\left(t_{i}-\tau_{i j}\right)} \\
\leq & \bar{r}_{i}+\sum_{j=1, j \neq i}^{n} \bar{a}_{i j} e^{\alpha_{i j} x_{j}\left(t_{j}\right)}+\sum_{j=1, j \neq i}^{n} \bar{b}_{i j} e^{\beta_{i j} x_{j}\left(t_{j}-\tau_{i j}\right)} \\
\leq & \bar{r}_{i}+\sum_{j=1, j \neq i}^{n} \bar{a}_{i j} e^{\alpha_{j j} x_{j}\left(t_{j}\right)}+\sum_{j=1, j \neq i}^{n} \bar{b}_{i j} e^{\alpha_{j j} x_{j}\left(t_{j}\right)} \\
= & \bar{r}_{i}+\sum_{j=1, j \neq i}^{n}\left(\bar{a}_{i j}+\bar{b}_{i j}\right) e^{\alpha_{j j} x_{j}\left(t_{j}\right)} \cdot
\end{aligned}
$$

Here we used $\left(\mathrm{H}_{2}\right)$. Letting $\left(\underline{a}_{i i}+\underline{b}_{i i}\right) e^{\alpha_{i i} x_{i}\left(t_{i}\right)}=z_{i}\left(t_{i}\right)$, it follows from (39) that

$$
z_{i}\left(t_{i}\right) \leq \bar{r}_{i}+\sum_{j=1, j \neq i}^{n}\left(\bar{a}_{i j}+\bar{b}_{i j}\right)\left(\underline{a}_{j j}+\underline{b}_{j j}\right)^{-1} z_{j}\left(t_{j}\right)
$$

or

$$
z_{i}\left(t_{i}\right)-\sum_{j=1, j \neq i}^{n} \frac{\bar{a}_{i j}+\bar{b}_{i j}}{\underline{a}_{j j}+\underline{b}_{j j}} z_{j}\left(t_{j}\right) \leq \bar{r}_{i}
$$

which implies

$$
\begin{aligned}
& \left(\begin{array}{cccc}
1 & -\frac{\bar{a}_{12}+\bar{b}_{12}}{\underline{a}_{22}+\underline{b}_{22}} & \ldots & -\frac{\bar{a}_{1 n}+\bar{b}_{1 n}}{\underline{a}_{n n}+\underline{b}_{n n}} \\
-\frac{\bar{a}_{21}+\bar{b}_{21}}{\underline{a}_{11}+\underline{b}_{11}} & 1 & \ldots & -\frac{\bar{a}_{2 n}+\bar{b}_{2 n}}{\underline{a}_{n n}+\underline{b}_{n n}} \\
\ldots & \ldots & \ldots & \ldots \\
-\frac{\bar{a}_{n 1}+\bar{b}_{n 1}}{\underline{a}_{11}+\underline{b}_{11}} & -\frac{\bar{a}_{n 2}+\bar{b}_{n 2}}{\underline{a}_{22}+\underline{b}_{22}} & \ldots & 1
\end{array}\right) \\
& \times\left(\begin{array}{c}
z_{1}\left(t_{1}\right) \\
z_{2}\left(t_{2}\right) \\
\cdots \\
z_{n}\left(t_{n}\right)
\end{array}\right) \leq\left(\begin{array}{c}
\bar{r}_{1} \\
\bar{r}_{2} \\
\cdots \\
\bar{r}_{n}
\end{array}\right) .
\end{aligned}
$$

Set $D=\left(D_{1}, D_{2}, \ldots, D_{n}\right)^{T}=\left(\bar{r}_{1}, \bar{r}_{2}, \ldots, \bar{r}_{n}\right)^{T}$. It follows from (42) that

$$
(E-\mathscr{K})\left(z_{1}\left(t_{1}\right), z_{2}\left(t_{2}\right), \ldots, z_{n}\left(t_{n}\right)\right)^{T} \leq D .
$$

In view of $\rho(\mathscr{K})<1$ and Lemma $5,\left(E_{n}-\mathscr{K}\right)^{-1} \geq 0$. Let

$$
H=\left(\widetilde{h}_{1}, \widetilde{h}_{2}, \ldots, \widetilde{h}_{n}\right)^{T}:=(E-\mathscr{K})^{-1} D \geq 0 .
$$

Then it follows from (43) and (44) that

$$
\begin{aligned}
& \left(z_{1}\left(t_{1}\right), z_{2}\left(t_{2}\right), \ldots, z_{n}\left(t_{n}\right)\right)^{T} \leq H, \\
& \text { or } z_{i}\left(t_{i}\right) \leq \widetilde{h}_{i}, \quad i=1,2, \ldots, n
\end{aligned}
$$

which implies

$$
\begin{array}{r}
\left|x_{i}(t)\right|_{0}=\max _{t \in[0, \omega]}\left|x_{i}(t)\right|=x_{i}\left(t_{i}\right) \leq \frac{1}{\alpha_{i i}} \ln \frac{\widetilde{h}_{i}}{\underline{a}_{i i}+\underline{b}_{i i}}, \\
i=1,2, \ldots, n .
\end{array}
$$

On the other hand, it follows from (44) that

$$
\begin{aligned}
& (E-\mathscr{K}) H=D, \quad \text { or } \quad H=\mathscr{K} H+D, \\
& \text { that is } \widetilde{h}_{i}=\sum_{j=1}^{n} \Gamma_{i j} \widetilde{h}_{j}+D_{i}, \quad i=1,2, \ldots, n .
\end{aligned}
$$

Estimating (2), by using (45) and (47), we have

$$
\begin{aligned}
& \left|\dot{x}_{i}(t)\right|_{0}=\lambda\left|r_{i}(t)-\sum_{j=1}^{n} a_{i j}(t) e^{\alpha_{i j} x_{j}(t)}-\sum_{j=1}^{n} b_{i j}(t) e^{\beta_{i j} x_{j}\left(t-\tau_{i j}\right)}\right|_{0} \\
& \leq \bar{r}_{i}+\sum_{j=1}^{n} \bar{a}_{i j}\left|e^{\alpha_{i j} x_{j}(t)}\right|_{0}+\sum_{j=1}^{n} \bar{b}_{i j}\left|e^{\beta_{i j} x_{j}\left(t-\tau_{i j}\right)}\right|_{0} \\
& \leq \bar{r}_{i}+\sum_{j=1}^{n} \bar{a}_{i j} e^{\alpha_{j j} x_{j}\left(t_{j}\right)}+\sum_{j=1}^{n} \bar{b}_{i j} e^{\alpha_{j j} x_{j}\left(t_{j}\right)} \\
& =\bar{r}_{i}+\sum_{j=1}^{n} \frac{\bar{a}_{i j}+\bar{b}_{i j}}{\underline{a}_{j j}+\underline{b}_{j j}} z_{j}\left(t_{j}\right) \\
& =\bar{r}_{i}+\sum_{j=1, j \neq i}^{n} \frac{\bar{a}_{i j}+\bar{b}_{i j}}{\underline{a}_{j j}+\underline{b}_{j j}} z_{j}\left(t_{j}\right)+\frac{\bar{a}_{i i}+\bar{b}_{i i}}{\underline{a}_{i i}+\underline{b}_{i i}} z_{i}\left(t_{i}\right) \\
& \leq D_{i}+\sum_{j=1}^{n} \Gamma_{i j} \widetilde{h}_{j}+\frac{\bar{a}_{i i}+\bar{b}_{i i}}{\underline{a}_{i i}+\underline{b}_{i i}} z_{i}\left(t_{i}\right) \\
& \leq \widetilde{h}_{i}+\frac{\bar{a}_{i i}+\bar{b}_{i i}}{\underline{a}_{i i}+\underline{b}_{i i}} \\
& =\left[1+\frac{\bar{a}_{i i}+\bar{b}_{i i}}{\underline{a}_{i i}+\underline{b}_{i i}}\right] \tilde{h}_{i} .
\end{aligned}
$$

We can choose a large enough real number $(d>1)$ such that

$$
\frac{1}{\alpha_{i i}} \ln \frac{d \tilde{h}_{i}}{\underline{a}_{i i}+\underline{b}_{i i}}>\frac{1}{\alpha_{i i}} \ln \frac{\tilde{h}_{i}}{\underline{a}_{i i}+\underline{b}_{i i}}+\left[1+\frac{\bar{a}_{i i}+\bar{b}_{i i}}{\underline{a}_{i i}+\underline{b}_{i i}}\right] \tilde{h}_{i} .
$$

Set $h_{i}=\left(1 / \alpha_{i i}\right) \ln \left(d \widetilde{h}_{i} /\left(\underline{a}_{i i}+\underline{b}_{i i}\right)\right)$. Then for any solution of $L x=\lambda N x$, we have

$$
\begin{aligned}
\left|x_{i}(t)\right|_{1} & =\left|x_{i}(t)\right|_{0}+\left|\dot{x}_{i}(t)\right|_{0} \\
& \leq \frac{1}{\alpha_{i i}} \ln \frac{\tilde{h}_{i}}{\underline{a}_{i i}+\underline{b}_{i i}}+\left[1+\frac{\bar{a}_{i i}+\bar{b}_{i i}}{\underline{a}_{i i}+\underline{b}_{i i}}\right] \widetilde{h}_{i}<h_{i},
\end{aligned}
$$


for all $i=1,2, \ldots, n$. Obviously, $h_{i}$ are independent of $\lambda$ and the choice of $x(t)$. Consequently, taking $h_{i}=$ $\left(1 / \alpha_{i i}\right) \ln \left(d \widetilde{h}_{i} /\left(\underline{a}_{i i}+\underline{b}_{i i}\right)\right)$, the open subset $\Omega$ satisfies that $L x \neq \lambda N x$ for each $\lambda \in(0,1), x \in \partial \Omega \cap \operatorname{Dom} L$; that is, the open subset $\Omega$ satisfies assumption (i) of Lemma 3.

Step 3. In what follows, we verify that for the given open bounded set $\Omega$, assumption (ii) of Lemma 3 also holds. That is, for each $x \in \partial \Omega \cap \operatorname{Ker} L, Q N x \neq 0$ and $\operatorname{deg}\{J Q N, \Omega \cap$ Ker $L, 0\} \neq 0$.

Take $x \in \partial \Omega \cap \operatorname{Ker} L$. Then, in view of $\operatorname{Ker} L=\mathbf{R}^{n}, x$ is a constant vector in $\mathbf{R}^{n}$, denoted by $x=\left(x_{1}, x_{2}, \ldots, x_{n}\right)^{T}$ and by the property

$$
\left|x_{i}\right|=\left|x_{i}\right|_{0}=\left|x_{i}\right|_{1}=h_{i}, \quad \forall i=1,2, \ldots, n .
$$

Operate $x$ by $Q N$, and we obtain that, for $i=1,2, \ldots, n$,

$$
\begin{array}{r}
(\mathrm{QNx})_{i}=m\left(r_{i}\right)-\sum_{j=1}^{n} m\left(a_{i j}\right) e^{\alpha_{i j} x_{j}}-\sum_{j=1}^{n} m\left(b_{i j}\right) e^{\beta_{i j} x_{j}}, \\
i=1,2, \ldots, n .
\end{array}
$$

We claim that $\left|(Q N x)_{i}\right|>0$, for $i=1,2, \ldots, n$. If this is not valid, suppose that there exists a certain $k \in\{1,2, \ldots, n\}$ such that $\left|(Q N x)_{k}\right|=0$; that is,

$$
m\left(r_{k}\right)-\sum_{j=1}^{n} m\left(a_{k j}\right) e^{\alpha_{k j} x_{j}}-\sum_{j=1}^{n} m\left(b_{k j}\right) e^{\beta_{k j} x_{j}}=0,
$$

or

$$
\begin{gathered}
m\left(a_{k k}\right) e^{\alpha_{k k} x_{k}}+m\left(b_{k k}\right) e^{\beta_{k k} x_{k}} \\
=m\left(r_{k}\right)-\sum_{j=1, j \neq k}^{n} m\left(a_{k j}\right) e^{\alpha_{k j} x_{j}} \\
\quad-\sum_{j=1, j \neq k}^{n} m\left(b_{k j}\right) e^{\beta_{k j} x_{j}} .
\end{gathered}
$$

That is,

$$
\begin{aligned}
{\left[m\left(a_{k k}\right)+m\left(b_{k k}\right)\right] e^{\alpha_{k k} x_{k}}=} & m\left(r_{k}\right)-\sum_{j=1, j \neq k}^{n} m\left(a_{k j}\right) e^{\alpha_{k j} x_{j}} \\
& -\sum_{j=1, j \neq k}^{n} m\left(b_{k j}\right) e^{\beta_{k j} x_{j}} .
\end{aligned}
$$

Letting $\left[m\left(a_{k k}\right)+m\left(b_{k k}\right)\right] e^{\alpha_{k k} x_{k}}=y_{k}$, we have

$$
\begin{gathered}
e^{\alpha_{k j} x_{j}}=\left(\frac{y_{j}}{m\left(a_{j j}\right)+m\left(b_{j j}\right)}\right)^{\alpha_{k j} / \alpha_{j j}}, \\
e^{\beta_{k j} x_{j}}=\left(\frac{y_{j}}{m\left(a_{j j}\right)+m\left(b_{j j}\right)}\right)^{\beta_{k j} / \alpha_{j j}}, \\
\alpha_{k j} \leq \alpha_{j j}, \quad \beta_{k j} \leq \alpha_{j j}, \quad \frac{y_{j}}{m\left(a_{j j}\right)}>1 .
\end{gathered}
$$

In view of (51), we get

$$
\begin{aligned}
\left|y_{i}\right| & =\left|y_{i}\right|_{0}=\left|y_{i}\right|_{1} \\
& =\left[m\left(a_{i i}\right)+m\left(b_{i i}\right)\right] e^{\alpha_{i i} h_{i}} \\
& =\left[m\left(a_{i i}\right)+m\left(b_{i i}\right)\right] e^{\ln \left(d \widetilde{h}_{i} /\left(a_{i i}+\underline{b}_{i i}\right)\right)} \\
& =\left[m\left(a_{i i}\right)+m\left(b_{i i}\right)\right] \frac{d \widetilde{h}_{i}}{\underline{a}_{i i}+\underline{b}_{i i}}, \quad \forall i=1,2, \ldots, n .
\end{aligned}
$$

Note that $f \leq m(f) \leq \bar{f}$. It follows from (2), (57), and (47) that

$$
\begin{aligned}
& d \widetilde{h}_{k} \leq\left[m\left(a_{k k}\right)+m\left(b_{k k}\right)\right] \frac{d \widetilde{h}_{k}}{\left(\underline{a}_{k k}+\underline{b}_{k k}\right)}=\left|y_{k}\right| \\
& \leq\left|m\left(r_{k}\right)-\sum_{j=1, j \neq k}^{n} m\left(a_{k j}\right) e^{\alpha_{k j} x_{j}}-\sum_{j=1, j \neq k}^{n} m\left(b_{k j}\right) e^{\beta_{k j} x_{j}}\right| \\
& \leq m\left(r_{k}\right)+\sum_{j=1, j \neq k}^{n} m\left(a_{k j}\right)\left(\frac{\left|y_{j}\right|}{m\left(a_{j j}\right)+m\left(b_{j j}\right)}\right)^{\alpha_{k j} / \alpha_{j j}} \\
& +\sum_{j=1, j \neq k}^{n} m\left(b_{k j}\right)\left(\frac{\left|y_{j}\right|}{m\left(a_{j j}\right)+m\left(b_{j j}\right)}\right)^{\beta_{k j} / \alpha_{j j}} \\
& \leq \overline{r_{k}}+\sum_{j=1, j \neq k}^{n} m\left(a_{k j}\right) \frac{\left|y_{j}\right|}{m\left(a_{j j}\right)+m\left(b_{j j}\right)} \\
& +\sum_{j=1, j \neq k}^{n} m\left(b_{k j}\right) \frac{\left|y_{j}\right|}{m\left(a_{j j}\right)+m\left(b_{j j}\right)} \\
& =\overline{r_{k}}+\sum_{j=1, j \neq k}^{n} \frac{m\left(a_{k j}\right)+m\left(b_{k j}\right)}{m\left(a_{j j}\right)+m\left(b_{j j}\right)}\left|y_{j}\right| \\
& \leq \overline{r_{k}}+\sum_{j=1, j \neq k}^{n} \frac{m\left(a_{k j}\right)+m\left(b_{k j}\right)}{m\left(a_{j j}\right)+m\left(b_{j j}\right)} \\
& \times\left[m\left(a_{j j}\right)+m\left(b_{j j}\right)\right] \frac{d \widetilde{h_{j}}}{\left(\underline{a}_{j j}+\underline{b}_{j j}\right)} \\
& \leq D_{k}+\sum_{j=1, j \neq k}^{n} \frac{\bar{a}_{k j}+\bar{b}_{k j}}{\underline{a}_{j j}+\underline{b}_{j j}} d \widetilde{h_{j}} \\
& <d D_{k}+d \sum_{j=1, j \neq k}^{n} \frac{\bar{a}_{k j}+\bar{b}_{k j}}{\underline{a}_{j j}+\underline{b}_{j j}} \\
& <d\left[D_{k}+\sum_{j=1, j \neq k}^{n} \Gamma_{k j} \widetilde{h_{j}}\right] \\
& =d \widetilde{h_{k}} \text {, }
\end{aligned}
$$


which is a contradiction. Therefore, for any $x \in \partial \Omega \cap \operatorname{Ker} L$, $\left|(Q N x)_{i}\right|>0$ for all $i=1,2, \ldots, n$. That is, $(Q N x) \neq 0$, for $x \in$ $\partial \Omega \cap \operatorname{Ker} L$. Furthermore, in view of $\left(\mathrm{H}_{1}\right)$ and Definition 2, it is easy to see that

$$
\operatorname{deg}\{J Q N, \Omega \cap \operatorname{Ker} L, 0\} \neq 0
$$

where $\operatorname{deg}(\cdot)$ is the Brouwer degree and $J$ is the identity mapping since $\operatorname{Im} Q=\operatorname{Ker} L$.

So far, we have shown that the open subset $\Omega \subset X$ satisfies all the assumptions of Lemma 3. Hence, by Lemma 3, system (14) has at least one positive $\omega$-periodic solution in $\operatorname{Dom} L \cap \bar{\Omega}$. By (13), system (3) has at least one positive $\omega$ periodic solution, denoted by $\tilde{y}(t)$. This completes the proof of Theorem 6.

\section{Globally Asymptotic Stability}

Under the assumption of Theorem 6, we know that system (3) has at least one positive $\omega$-periodic solution, denoted by $\widetilde{y}(t)=\left(\tilde{y}_{1}(t), \ldots, \tilde{y}_{n}(t)\right)^{T}$. The aim of this section is to derive a set of sufficient conditions which guarantee the global asymptotic stability of the positive $\omega$-periodic solution $y^{*}(t)$. As pointed out in Section 1, because $y_{j}(t)$ has been changed to $y_{j}^{\alpha_{i j}}(t)$ in (3), the previous method in Xia and Han [8] cannot be applied to study the stability of system (3) directly. Before the formal analysis, we recall some facts which will be used in the proof.

Lemma 7 (see [21]). Let $f$ be a nonnegative function defined on $[0,+\infty]$ such that $f$ is integrable on $[0,+\infty]$ and is uniformly continuous on $[0,+\infty]$. Then $\lim _{t \rightarrow+\infty} f(t)=0$.

Lemma 8 (see $[19,20])$. Let $\mathbb{Q}=\left(q_{i j}\right)_{n \times n}$ be a matrix with nonpositive off-diagonal elements. $Q$ is an $M$-matrix if and only if there exists a positive diagonal matrix $\Xi=\operatorname{diag}\left(\xi_{1}, \xi_{2} \ldots, \xi_{n}\right)$ such that

$$
\xi_{i} q_{i i}>\sum_{j \neq i} \xi_{j} q_{j i}, \quad i=1,2, \ldots, n .
$$

We need a lemma which can follow immediately from Theorem 2.1 in Xia et al. [22]. We consider the following logistic equation:

$$
\dot{x}_{i}(t)=x_{i}(t)\left[r_{i}(t)-a_{i i}(t) x_{i}^{\alpha_{i i}}(t)\right] .
$$

Since $r_{i}(t)$ is nonnegative and $a_{i i}(t)$ are strictly positive, it follows immediately from Lemma 2.1 in Xia et al. [22] that system (61) has a unique positive solution, denoted by $X_{i}(t)$, with $X_{i}(t) \leq\left(\bar{r}_{i} / \underline{a}_{i i}\right)^{1 / \alpha_{i i}}$, which is globally asymptotically stable. Then, as a special case of Theorem 2.1 in [22], we have the following.

\section{Lemma 9. Suppose that}

$$
\left(\mathrm{H}_{4}\right) m\left(r_{i}(t)-\sum_{j=1, j \neq i}^{n}\left[a_{i j}(t)+b_{i j}\left(t+\tau_{i j}\right)\right] X_{j}^{\alpha_{i j}}(t)\right)>0 ;
$$
then system (3) is bounded above and below.
Lemma 10 (see [23]). If $x, y>1$, and $\alpha_{j i} \leq \alpha_{i i},(i, j=$ $1,2, \ldots, n)$, then

$$
\left|x^{\alpha_{j i}}-y^{\alpha_{j i}}\right| \leq\left|x^{\alpha_{i i}}-y^{\alpha_{i i}}\right|, \quad i, j=1,2, \ldots, n .
$$

Theorem 11. Assume that $\alpha_{i j}=\beta_{i j}$; if $\left(H_{1}\right)-\left(H_{4}\right)$ hold, then system (3) has a unique positive $\omega$-periodic solution $\widetilde{y}(t)$ which is globally asymptotically stable.

Proof. By Lemma 9, system (3) is bounded below. Thus there exist positive constants $m_{i}>0$ such that $y_{i}(t) \geq m_{i}$. We proceed the proof of this theorem with two steps.

Step 1. Choose positive constants $d_{i}\left(0<d_{i}<m_{i}\right), i=1$, $2, \ldots, n$, such that $\rho(\mathscr{K})=\rho(\mathscr{H})$, where $\mathscr{H}=\left(h_{i j}\right)_{n \times n}$ and

$$
h_{i j}= \begin{cases}0, & i=j, \\ \left(\underline{a}_{j j}+\underline{b}_{j j}\right)^{-1}\left(\bar{a}_{i j}+\bar{b}_{i j}\right) d_{j}^{\alpha_{i j}-\alpha_{j j},}, & i \neq j .\end{cases}
$$

We claim that the positive constants $d_{i}$ can be definitely chosen. By similar arguments in [23], one can prove this fact.

Step 2. Prove the global asymptotic stability of system (3). Changing variables $w_{i}=y_{i} / d_{i}$, by noticing that $\beta_{i j}=\alpha_{i j}$, then system (3) changes to

$$
\begin{array}{r}
\dot{w}_{i}(t)=w_{i}(t)\left[r_{i}(t)-\sum_{j=1}^{n} a_{i j}(t) d_{j}^{\alpha_{i j}} w_{j}^{\alpha_{i j}}(t)\right. \\
\left.-\sum_{j=1}^{n} b_{i j}(t) d_{j}^{\alpha_{i j}} w_{j}^{\alpha_{i j}}\left(t-\tau_{i j}\right)\right], \\
i=1,2, \ldots, n .
\end{array}
$$

In view of $\left(\mathrm{H}_{1}\right)-\left(\mathrm{H}_{3}\right)$, system (3) has at least a positive periodic solution. By the linear transformation $w_{i}=y_{i} / d_{i}$, we know that system (64) also has at least a positive periodic solution, denoted by $\widetilde{w}(t)=\left(\widetilde{w}_{1}(t), \ldots, \widetilde{w}_{n}(t)\right)$. In order to show the global asymptotic stability of system (3), it suffices to show that $\widetilde{w}(t)$ of system (64) is globally asymptotically stable. For this purpose, let $w(t)=\left(w_{1}(t), \ldots, w_{n}(t)\right)$ be any other positive solution of system (64). And we define a Lyapunov functional $V(t)$ as follows:

$$
\begin{aligned}
& V(t)=\sum_{i=1}^{n} \xi_{i}\left[\left|\ln w_{i}(t)-\ln \widetilde{w}_{i}(t)\right|\right. \\
&+\sum_{j=1, j \neq i}^{n} \int_{t-\tau_{i j}}^{t} b_{i j}\left(s+\tau_{i j}\right) d_{j}^{\alpha^{i j}} \\
&\left.\quad \times\left|w_{j}^{\alpha_{i j}}(s)-\widetilde{w}_{j}^{\alpha_{i j}}(s)\right| d s\right] .
\end{aligned}
$$


Calculating the upper right derivative of $V(t)$ along (64), it follows from (65) that

$D^{+} V(t)$

$=\sum_{i=1}^{n} \xi_{i} \operatorname{sgn}\left|\ln w_{i}(t)-\ln \widetilde{w}_{i}(t)\right|$

$\times\left[\frac{w_{i}^{\prime}(t)}{w_{i}(t)}-\frac{\widetilde{w}_{i}^{\prime}(t)}{\widetilde{w}_{i}(t)}\right.$

$+\sum_{j=1, j \neq i}^{n} b_{i j}\left(t+\tau_{i j}\right) d_{j}^{\alpha_{i j}}\left|w_{j}^{\alpha_{i j}}(t)-\widetilde{w}_{j}^{\alpha_{i j}}(t)\right|$

$\left.-\sum_{j=1, j \neq i}^{n} b_{i j}(t) d_{j}^{\alpha_{i j}}\left|w_{j}^{\alpha_{i j}}\left(t-\tau_{i j}\right)-\widetilde{w}_{j}^{\alpha_{i j}}\left(t-\tau_{i j}\right)\right|\right]$

$=\sum_{i=1}^{n} \xi_{i} \frac{\ln w_{i}(t)-\ln \widetilde{w}_{i}(t)}{\left|\ln w_{i}(t)-\ln \widetilde{w}_{i}(t)\right|}$

$\times\left[-\sum_{j=1}^{n} a_{i j}(t) d_{j}^{\alpha_{i j}}\left(w_{j}^{\alpha_{i j}}(t)-\widetilde{w}_{j}^{\alpha_{i j}}(t)\right)\right.$

$-b_{i i}(t) d_{j}^{\alpha_{i i}}\left(w_{i}^{\alpha_{i i}}(t)-\widetilde{w}_{i}^{\alpha_{i i}}(t)\right)$

$\left.+\sum_{j=1, j \neq i}^{n} b_{i j}\left(t+\tau_{i j}\right) d_{j}^{\alpha_{i j}}\left|w_{j}^{\alpha_{i j}}(t)-\widetilde{w}_{j}^{\alpha_{i j}}(t)\right|\right]$

$+\sum_{i=1}^{n} \sum_{j=1, j \neq i}^{n} \xi_{i} b_{i j}\left(t+\tau_{i j}\right) d_{j}^{\alpha_{i j}}\left|w_{j}^{\alpha_{i j}}(t)-\widetilde{w}_{j}^{\alpha_{i j}}(t)\right|$

$\leq-\sum_{i=1}^{n} \xi_{i} a_{i i}(t) d_{i}^{\alpha_{i i}}\left|w_{i}^{\alpha_{i i}}(t)-\widetilde{w}_{i}^{\alpha_{i i}}(t)\right|$

$+\sum_{i=1}^{n} \sum_{j=1, j \neq i}^{n} \xi_{j} a_{j i}(t) d_{i}^{\alpha_{j i}}\left|w_{i}^{\alpha_{j i}}(t)-\widetilde{w}_{i}^{\alpha_{j i}}(t)\right|$

$-\sum_{i=1}^{n} \xi_{i} b_{i i}(t) d_{i}^{\alpha_{i i}}\left(w_{i}^{\alpha_{i i}}(t)-\widetilde{w}_{i}^{\alpha_{i i}}(t)\right)$

$+\sum_{i=1}^{n} \sum_{j=1, j \neq i}^{n} \xi_{j} b_{j i}\left(t+\tau_{j i}\right) d_{i}^{\alpha_{j i}}\left|w_{i}^{\alpha_{j i}}(t)-\widetilde{w}_{i}^{\alpha_{j i}}(t)\right|$

$\leq-\sum_{i=1}^{n} \xi_{i}\left[a_{i i}(t)+b_{i i}(t)\right] d_{i}^{\alpha_{i i}}\left|w_{i}^{\alpha_{i i}}(t)-\widetilde{w}_{i}^{\alpha_{i i}}(t)\right|$

$+\sum_{i=1}^{n} \sum_{j=1, j \neq i}^{n} \xi_{j} a_{j i}(t) d_{i}^{\alpha_{j i}}\left|w_{i}^{\alpha_{j i}}(t)-\widetilde{w}_{i}^{\alpha_{j i}}(t)\right|$

$+\sum_{i=1}^{n} \sum_{j=1, j \neq i}^{n} \xi_{j} b_{j i}\left(t+\tau_{j i}\right) d_{i}^{\alpha_{j i}}\left|w_{i}^{\alpha_{j i}}(t)-\widetilde{w}_{i}^{\alpha_{j i}}(t)\right|$.

(66)
Note that $w_{i}(t), \widetilde{w}_{i}(t) \geq m_{i} / d_{i}>1$ and $\alpha_{j i} \leq \alpha_{i i}$. By Lemma 10, we have

$$
\left|w_{i}^{\alpha_{j i}}(t)-\widetilde{w}_{i}^{\alpha_{j i}}(t)\right| \leq\left|w_{i}^{\alpha_{i i}}(t)-\widetilde{w}_{i}^{\alpha_{i i}}(t)\right| .
$$

It follows from (66) and (67) that

$$
\begin{aligned}
D^{+} V(t) \leq & -\sum_{i=1}^{n} \xi_{i}\left[a_{i i}(t)+b_{i i}(t)\right] d_{i}^{\alpha_{i i}}\left|w_{i}^{\alpha_{i i}}(t)-\widetilde{w}_{i}^{\alpha_{i i}}(t)\right| \\
& +\sum_{i=1}^{n} \sum_{j=1, j \neq i}^{n} \xi_{j} a_{j i}(t) d_{i}^{\alpha_{j i}}\left|w_{i}^{\alpha_{i i}}(t)-\widetilde{w}_{i}^{\alpha_{i i}}(t)\right| \\
& +\sum_{i=1}^{n} \sum_{j=1, j \neq i}^{n} \xi_{j} b_{j i}\left(t+\tau_{j i}\right) d_{i}^{\alpha_{j i}}\left|w_{i}^{\alpha_{i i}}(t)-\widetilde{w}_{i}^{\alpha_{i i}}(t)\right| \\
\leq & -\sum_{i=1}^{n}\left[\xi_{i}\left(\underline{a}_{i i}+\underline{b}_{i i}\right) d_{i}^{\alpha_{i i}}\right. \\
& \left.\quad-\sum_{j=1, j \neq i}^{n} \xi_{j} d_{i}^{\alpha_{j i}}\left(\bar{a}_{j i}+\bar{b}_{j i}\right)\right] \\
& \times\left|w_{i}^{\alpha_{i i}}(t)-\widetilde{w}_{i}^{\alpha_{i i}}(t)\right| .
\end{aligned}
$$

From Step 1, we know $\rho\left(\mathscr{H}^{T}\right)=\rho(\mathscr{H})=\rho(\mathscr{K})<1$. Thus, in view of Lemma 5 and Definition $4,\left(E-\mathscr{H}^{T}\right)$ is an $M$-matrix, where $E$ denotes an identity matrix of size $n$. Therefore, by Lemma 8 , there exists a diagonal matrix $\Xi=\operatorname{diag}\left(\xi_{1}, \ldots, \xi_{n}\right)$ with positive diagonal elements such that the product $(E-$ $\left.\mathscr{H}^{T}\right) \Xi$ is strictly diagonally dominant with positive diagonal entries; namely,

$$
\begin{array}{r}
\xi_{i}>\sum_{j=1, j \neq i}^{n} \xi_{j} h_{j i}, \\
\text { or } \xi_{i} d_{i}^{\alpha_{i i}}\left(\underline{a}_{i i}+\underline{b}_{i i}\right)-\sum_{j=1, j \neq i}^{n} \xi_{j} d_{i}^{\alpha_{j i}}\left(\bar{a}_{j i}+\bar{b}_{j i}\right)>0, \\
i=1, \ldots, n .
\end{array}
$$

It follows from (68) and (69) that $D^{+} V(t) \leq 0$. Obviously, the zero solution of (64) is Lyapunov stable. On the other hand, integrating (69) over $\left[t_{0}, t\right]$ leads to

$$
V(t)-V\left(t_{0}\right) \leqslant-k \int_{t_{0}}^{t} \sum_{i=1}^{n}\left|w_{i}^{\alpha_{i i}}(s)-\widetilde{w}_{i}^{\alpha_{i i}}(s)\right| d s, \quad t \geq t_{0},
$$

or

$$
V(t)+k \int_{t_{0}}^{t} \sum_{i=1}^{n}\left|w_{i}^{\alpha_{i i}}(s)-\widetilde{w}_{i}^{\alpha_{i i}}(s)\right| d s \leq V\left(t_{0}\right)<+\infty, \quad t \geqslant t_{0} .
$$

Noting that $V(t) \geqslant 0$, it follows that

$$
\int_{t_{0}}^{t} \sum_{i=1}^{n}\left|w_{i}^{\alpha_{i i}}(s)-\widetilde{w}_{i}^{\alpha_{i i}}(s)\right| d s \leq \frac{V\left(t_{0}\right)}{k}<+\infty, \quad t \geqslant t_{0} .
$$


Therefore, by Lemma 7, it is not difficult to conclude that

$$
\lim _{t \rightarrow+\infty}\left|w_{i}(t)-\widetilde{w}_{i}(t)\right|=0
$$

Which implies the global asymptotical stability of system (64). By the linear transformation $y_{i}(t)=d_{i} w_{i}(t), i=$ $1,2, \ldots, n$, the positive periodic solution $\tilde{y}(t)$ of (3) is also globally asymptotically stable. This completes the proof of Theorem 11.

\section{Remark and Discussion}

To illustrate the generality of our results, we will give a corollary in this section. Now recall that, for a given matrix $\mathscr{K}$, its spectral radius $\rho(\mathscr{K})$ is equal to the minimum of all matrix norms of $\mathscr{K}$; that is, for any matrix norm $\|\cdot\|, \rho(\mathscr{K}) \leq$ $\|\mathscr{K}\|$. Therefore, a corollary of Theorem 11 is stated as follows.

Corollary 12. In addition to $\left(\mathrm{H}_{1}\right),\left(\mathrm{H}_{2}\right)$, and $\left(\mathrm{H}_{4}\right)$, if one further supposes that there exist positive constants $\xi_{i}, i=$ $1,2, \ldots, n$, such that one of the following inequalities holds:

(1) $\max _{1 \leq j \leq n} \sum_{i=1, i \neq j}^{n}\left(\xi_{i}\left(\bar{a}_{i j}+\bar{b}_{i j}\right) / \xi_{j}\left(\underline{a}_{j j}+\underline{b}_{j j}\right)\right)<1$, or equivalently, $\left(\underline{a}_{j j}+\underline{b}_{j j}\right) \xi_{j}>\sum_{i=1, i \neq j}^{n}\left(\bar{a}_{i j}+\bar{b}_{i j}\right) \xi_{i}$ for all $j=1,2, \ldots, n$,

(2) $\sum_{i=1}^{n} \sum_{j=1}^{n}\left(\xi_{i}^{-1} \xi_{j} \Gamma_{i j}\right)^{2}<1$, where $\Gamma_{i j}$ has been defined in Theorem 6,

(3) $\max _{1 \leq i \leq n} \sum_{j=1, j \neq i}^{n}\left(\xi_{j}\left(\bar{a}_{j i}+\bar{b}_{j i}\right) / \xi_{i}\left(\underline{a}_{i i}+\underline{b}_{i i}\right)\right)<1$, or equivalently, $\left(\underline{a}_{i i}+\underline{b}_{i i}\right) \xi_{i}>\sum_{j=1, j \neq i}^{n}\left(\bar{a}_{j i}+\bar{b}_{j i}\right) \xi_{i}$ for all $i=1,2, \ldots, n$,

then system (3) has a unique positive w-periodic solution which is globally asymptotically stable.

Proof. For any matrix norm $\|\cdot\|$ and any nonsingular matrix $S,\|\mathscr{K}\|_{S}=\left\|S^{-1} \mathscr{K} S\right\|$ also defines a matrix norm. Let $\Xi=$ $\operatorname{diag}\left(\xi_{1}, \xi_{2}, \ldots, \xi_{n}\right)$. Then conditions (1)-(2) correspond to the column norms and Frobenius norm of matrix $\Xi \mathscr{K} \Xi^{-1}$, respectively. Condition (3) corresponds to the row norms of $\Xi \mathscr{K}^{T} \Xi^{-1}$ and note that $\rho\left(\Xi \mathscr{K}^{T} \Xi^{-1}\right)=\rho\left(\Xi \mathscr{K} \Xi^{-1}\right)$. Corollary 12 follows immediately.

Remark 13. The corollary implies that the conditions given in terms of the spectral radius are much better than the classic norms.

Now we consider a special case of system (3). Take $\alpha_{i j}=1$, $b_{i j}=0$, and $\tau_{i j} \equiv 0$; then system (3) reduces to the classical LV competition system which has been well studied in Xia and Han [8]:

$$
\dot{y}_{i}(t)=y_{i}(t)\left[r_{i}(t)-\sum_{j=1}^{n} a_{i j}(t) y_{j}(t)\right], \quad i=1,2, \ldots, n .
$$

Remark 14 . In this case, Theorem 11 and Corollary 12 reduce to the main results in Xia and Han [8].

\section{Discussion}

As we know, dynamic systems are often classified into two categories of either continuous-time or discrete-time systems. However, many real-world phenomena are neither purely continuous-time nor purely discrete-time. This leads to the development of dynamic systems with impulses, which display a combination of characteristics of both the continuoustime and discrete-time systems and hence provide a more natural framework for mathematical modeling of many realworld phenomena. Whether the new method proposed in this paper can be applied to study the existence and global asymptotic stability of the LV systems with impulses remains open.

\section{Examples}

In this section, some examples and their simulations are presented to illustrate the feasibility and effectiveness of our results.

Example 15. Consider the two-species competitive system

$$
\begin{array}{r}
\dot{y}_{1}(t)=y_{1}(t)\left[4-(2+\sin t) y_{1}^{2}(t)-\frac{1}{2} y_{2}(t)\right. \\
\left.-\frac{1}{8}(3+\cos t) y_{1}^{2}(t)-\frac{1}{2} y_{2}(t-0.2)\right], \\
\dot{y}_{2}(t)=y_{2}(t)\left[2-(1+\sin t) y_{1}(t)-(4-\cos t) y_{2}^{2}(t)\right. \\
\left.-\frac{1}{4}(1-\cos t) y_{1}(t-0.5)-\frac{1}{2} y_{2}^{2}(t)\right] .
\end{array}
$$

Corresponding to system (3), we have $\underline{a}_{11}=\bar{a}_{21}=2, \bar{a}_{12}=$ $\bar{b}_{12}=\bar{b}_{21}=\underline{b}_{22}=1 / 2, \bar{a}_{12}=1 / 2, \underline{a}_{22}=3, \underline{b}_{11}=1 / 4$. Simple computation shows

$$
\begin{aligned}
& \mathscr{K}=\left(\begin{array}{cc}
0 & \frac{\bar{a}_{12}+\bar{b}_{12}}{a_{22}+\underline{b}_{22}} \\
\bar{a}_{21}+\bar{b}_{21} & 0 \\
\underline{a}_{11}+\underline{b}_{11} &
\end{array}\right) \\
& =\left(\begin{array}{cc}
0 & \left(\frac{1}{2}+\frac{1}{2}\right) \times \frac{2}{7} \\
\left(2+\frac{1}{2}\right) \times \frac{4}{5} & 0
\end{array}\right) \\
& =\left(\begin{array}{ll}
0 & \frac{2}{7} \\
2 & 0
\end{array}\right) \text {. }
\end{aligned}
$$

And $\rho(\mathscr{K})=\sqrt{4 / 7}<1$. Thus, by Theorem 11, system (75) has a unique positive equilibrium which is globally asymptotically stable.

Remark 16. In this example, one can observe that, though the spectral $\rho(\mathscr{K})<1$, the matrix norms (including the row 
norm, the column norm, and the Frobenius norm) of matrix $\mathscr{K}$ may be bigger than 1 . For instance, the column norm

$$
\|\mathscr{K}\|_{1}=0+2>1 .
$$

Example 17. Consider the three-species competitive system

$$
\begin{aligned}
\dot{y}_{1}(t)=y_{1}(t)[ & -\frac{1}{2}(2+\sin t) y_{1}^{2}(t) \\
& -\frac{1}{8}(1+\cos t) y_{2}(t)-\frac{1}{12} y_{3}(t) \\
& -(2+\sin t) y_{1}^{2}(t)-\frac{1}{8}(1+\cos t) y_{2}(t-0.5) \\
& \left.-\frac{1}{4} y_{3}(t-0.2)\right], \\
\dot{y}_{2}(t)=y_{2}(t)\left[\begin{array}{l}
4 \\
-
\end{array}\right. & \frac{1}{8}(1-\sin t) y_{1}(t) \\
& -\frac{1}{2}(2+\sin t) y_{2}^{2}(t) \\
& -\frac{1}{8}(1-\sin t) y_{1}(t-0.5) \\
& \left.-(2+\sin t) y_{2}^{2}(t)\right], \\
& -\frac{1}{8}(1+\sin t) y_{2}(t)-\frac{1}{2}(2+\cos t) y_{3}^{2}(t) \\
\dot{y}_{3}(t)=y_{3}(t)\left[\begin{array}{l}
6 \\
-
\end{array}\right. & (1-\sin t) y_{1}(t) \\
& \left.-(2+\cos t) y_{3}^{2}(t)\right] . \frac{1}{4} y_{2}(t-0.2) \\
& \\
&
\end{aligned}
$$

Corresponding to system (3), we have $\underline{a}_{11}=\underline{a}_{22}=\underline{a}_{33}=1 / 2$, $\bar{a}_{12}=\bar{a}_{21}=\bar{a}_{32}=1 / 4, \bar{a}_{13}=1 / 12, \bar{a}_{23}=0, \bar{a}_{31}=2, \underline{b}_{11}=$ $\underline{b}_{22}=\underline{b}_{33}=\bar{b}_{31}=1, \bar{b}_{12}=\bar{b}_{13}=\bar{b}_{21}=\bar{b}_{32}=1 / 4$, and $\bar{b}_{23}=0$. Then, simple computation leads to

$$
\begin{aligned}
\mathscr{K} & =\left(\begin{array}{ccc}
0 & \frac{\bar{a}_{12}+\bar{b}_{12}}{\underline{a}_{22}+\underline{b}_{22}} & \frac{\bar{a}_{13}+\bar{b}_{13}}{\underline{a}_{33}+\underline{b}_{33}} \\
\frac{\bar{a}_{21}+\bar{b}_{21}}{\underline{a}_{11}+\underline{b}_{11}} & 0 & 0 \\
\frac{\bar{a}_{31}+\bar{b}_{31}}{\underline{a}_{11}+\underline{b}_{11}} & \frac{\bar{a}_{32}+\bar{b}_{32}}{\underline{a}_{22}+\underline{b}_{22}} & 0
\end{array}\right) \\
& =\left(\begin{array}{ccc}
0 & \frac{1}{3} & \frac{2}{9} \\
\frac{1}{3} & 0 & 0 \\
2 & \frac{1}{3} & 0
\end{array}\right) .
\end{aligned}
$$

Hence, by using MATLAB, we get

$$
\rho(\mathscr{K})=\max . \text { eigenvalues }[\mathscr{K}]=0.7667<1 .
$$

Thus, by Theorem 11, system (78) has a unique positive equilibrium which is globally asymptotically stable.

Remark 18. In this example, one can observe that though the spectral $\rho(\mathscr{K})<1$, the matrix norms (including the row norm, the column norm, and the Frobenius norm) of matrix $\mathscr{K}$ may be bigger than 1 . For instance, the column norm

$$
\|\mathscr{K}\|_{1}=0+\frac{1}{3}+2>1
$$

\section{Conflict of Interests}

The authors declare that there is no conflict of interests regarding the publication of this paper.

\section{Acknowledgments}

This work is supported by the National Natural Science Foundation of China under Grant no. 11271333 and ZJNSFC.

\section{References}

[1] Y. L. Song and S. L. Yuan, "Bifurcation analysis for a regulated logistic growth model," Applied Mathematical Modelling, vol. 31, no. 9, pp. 1729-1738, 2007.

[2] Y. L. Song, S. L. Yuan, and J. Zhang, "Bifurcation analysis in the delayed Leslie-Gower predator-prey system," Applied Mathematical Modelling, vol. 33, no. 11, pp. 4049-4061, 2009.

[3] S. L. Yuan and F. Zhang, "Stability and global Hopf bifurcation in a delayed predator-prey system," Nonlinear Analysis. Real World Applications, vol. 11, no. 2, pp. 959-977, 2010.

[4] M. Fan, K. Wang, and D. Jiang, "Existence and global attractivity of positive periodic solutions of periodic $n$-species LotkaVolterra competition systems with several deviating arguments," Mathematical Biosciences, vol. 160, no. 1, pp. 47-61, 1999.

[5] W. Ding and M. Han, "Dynamic of a non-autonomous predatorprey system with infinite delay and diffusion," Computers \& Mathematics with Application, vol. 56, no. 5, pp. 1335-1350, 2008.

[6] Y.-H. Xia, "Global analysis of an impulsive delayed LotkaVolterra competition system," Communications in Nonlinear Science and Numerical Simulation, vol. 16, no. 3, pp. 1597-1616, 2011.

[7] Y.-H. Xia, "Global asymptotic stability of an almost periodic nonlinear ecological model," Communications in Nonlinear Science and Numerical Simulation, vol. 16, no. 11, pp. 4451-4478, 2011.

[8] Y.-H. Xia and M. Han, "New conditions on the existence and stability of periodic solution in Lotka-Volterra's population system," SIAM Journal on Applied Mathematics, vol. 69, no. 6, pp. 1580-1597, 2009.

[9] K. Hong and P. Weng, "Stability and traveling waves of diffusive predator-prey model with age-structure and nonlocal effect," The Journal of Applied Analysis and Computation, vol. 2, no. 2, pp. 173-192, 2012. 
[10] I. Djellit, M. L. Sahari, and A. Hachemi, "Complex dynamics in 2-species predator-prey systems," The Journal of Applied Analysis and Computation, vol. 3, no. 1, pp. 11-20, 2013.

[11] M. E. Gilpin and F. J. Ayala, "Global models of growth and competition," Proceedings of the National Academy of Sciences of the United States of America, vol. 70, pp. 3590-3593, 1973.

[12] F. J. Ayala, M. E. Gilpin, and J. G. Ehrenfeld, "Competition between species: theoretical models and experimental tests," Theoretical Population Biology, vol. 4, pp. 331-356, 1973.

[13] F. D. Chen, "Average conditions for permanence and extinction in nonautonomous Gilpin-Ayala competition model," Nonlinear Analysis. Real World Applications, vol. 7, no. 4, pp. 895-915, 2006.

[14] R. M. May, "Time delay versus stability in population models with two or three trophic levels," Ecology, vol. 54, no. 2, pp. 315325, 1973.

[15] K. L. Cooke and Z. Grossman, "Discrete delay, distributed delay and stability switches," Journal of Mathematical Analysis and Applications, vol. 86, no. 2, pp. 592-627, 1982.

[16] W. Wendi and M. Zhien, "Harmless delays for uniform persistence," Journal of Mathematical Analysis and Applications, vol. 158, no. 1, pp. 256-268, 1991.

[17] R. E. Gaines and J. L. Mawhin, Coincidence Degree and Nonlinear Differential Equations, Springer, Berlin, Germany, 1977.

[18] D. Guo, J. Sun, and Z. Liu, Functional Method in Nonlinear Ordinary Differential Equations, Shangdong Scientific Press, Shandong, China, 2005.

[19] J. P. LaSalle, The Stability of Dynamical System, Society for Industrial and Applied Mathematics, Philadelphia, Pa, USA, 1976.

[20] A. Berman and R. J. Plemmons, Nonnegative Matrices in the Mathematical Science, Academic Press, New York, NY, USA, 1979.

[21] I. Barbălat, "Systems d'equations differentielle d'oscillations nonlineaires," Revue Roumaine de Mathématique Pures et Appliquées, vol. 4, pp. 267-270, 1959.

[22] Y.-H. Xia, M. Han, and Z. K. Huang, "Global attractivity of an almost periodic $N$-species nonlinear ecological competitive model," Journal of Mathematical Analysis and Applications, vol. 337, no. 1, pp. 144-168, 2008.

[23] Y.-H. Xia, "Periodic solution of certain nonlinear differential equations: via topological degree theory and matrix spectral theory," International Journal of Bifurcation and Chaos in Applied Sciences and Engineering, vol. 22, no. 8, Article ID 1250196, 17 pages, 2012. 


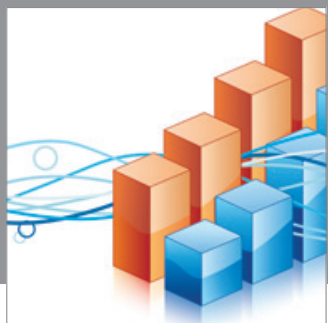

Advances in

Operations Research

mansans

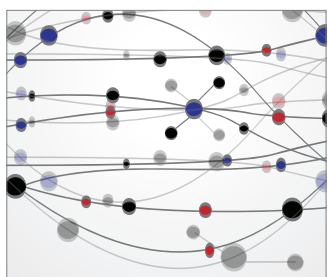

The Scientific World Journal
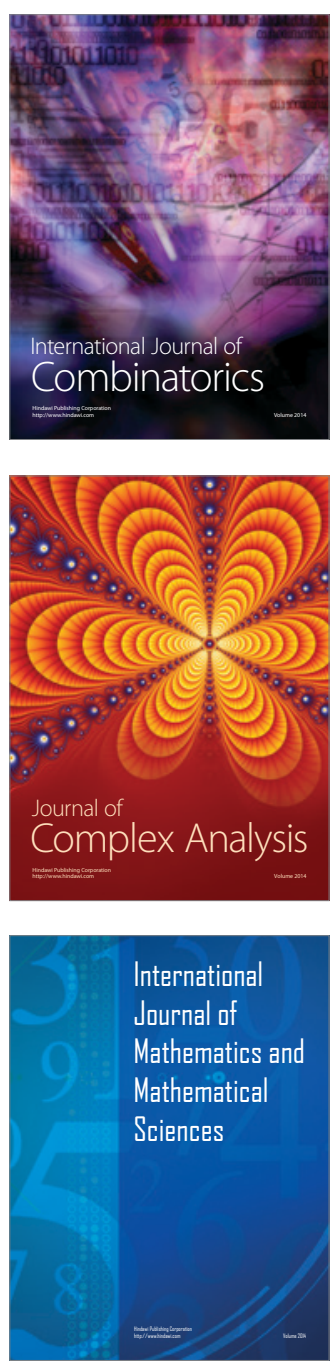
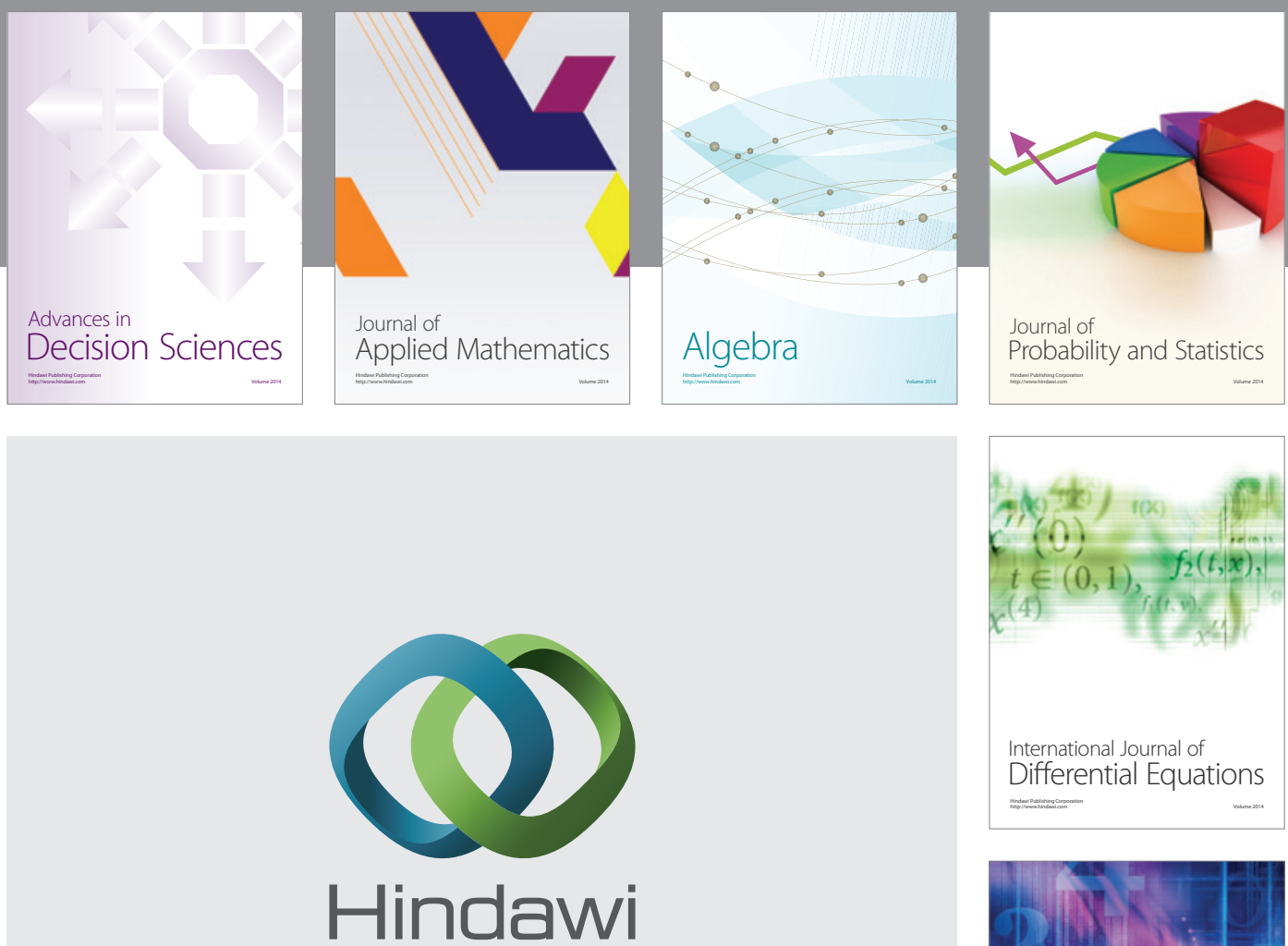

Submit your manuscripts at http://www.hindawi.com
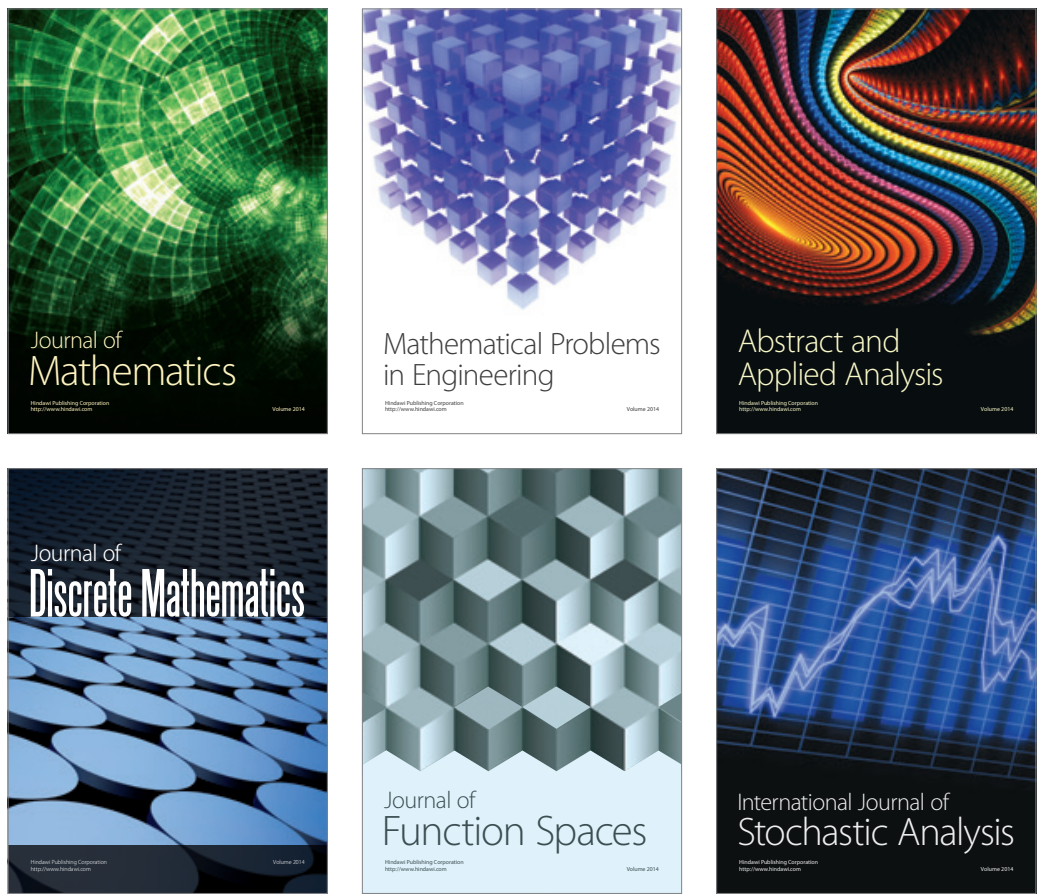

Journal of

Function Spaces

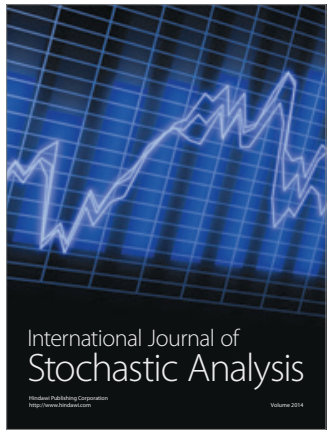

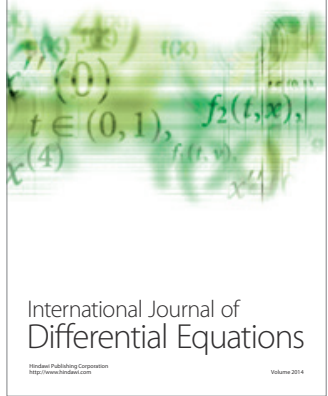
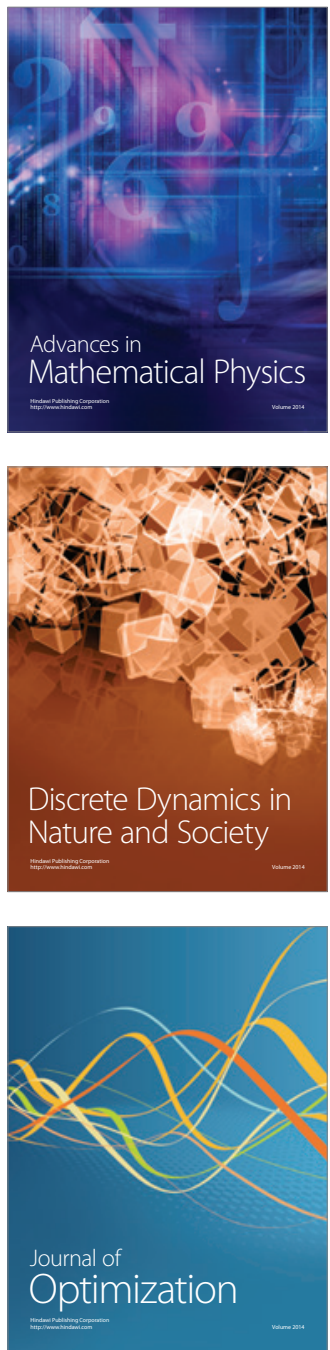